\title{
Direct imaging of ultrafast electron dynamics by X-ray sum frequency generation
}

\author{
Jérémy R. Rouxel ${ }^{1, *}$, Markus Kowalewski ${ }^{1,}$, Kochise Benett $^{1,}$, and Shaul Mukamel ${ }^{1, * *}$ \\ ${ }^{1}$ Department of Chemistry and Department of Physics and Astronomy, University of California,Irvine, \\ CA 92697, USA
}

\begin{abstract}
We present a nonlinear X-ray technique which provides a spatial electron density image of valence electron excitations. The technique combines a visible pump and an X-ray pulse and yields snapshots of the transition charge densities.
\end{abstract}

\section{Introduction}

$\mathrm{X}$-ray diffraction from molecules in the ground state produces an image of their charge density and time-resolved X-ray diffraction can monitor the motion of the nuclei. However, the density change of excited valence electrons upon optical excitation can barely be monitored with regular diffraction techniques due to the overwhelming dominant background contribution of the core electrons. We present a nonlinear X-ray technique made possible by novel free electron laser sources, which provides a spatial electron density image of valence electron excitations. The technique, sum frequency generation, combines a visible pump and a broadband X-ray diffraction pulse, yields snapshots of the transition charge densities, which represent the electron density variations upon optical excitation. Signals are illustrated by ab initio simulations of transition charge densities imaging for the optically induced electronic dynamics in a donor/acceptor substituted stilbene.

\section{Monitoring electronic dynamics via transition charge densities}

Time-resolved X-ray diffraction can provide stroboscopic snapshots of time-evolving excited state charge densities. The resulting real-time movies of the charge density can monitor the optically triggered evolution of the molecular geometry. Since optical excitations typically involve few valence electrons (e.g., excitation from the highest occupied to the lowest unoccupied molecular orbital), the ground and excited state charge densities are very similar and hard to distinguish by X-ray diffraction, which is dominated by the highly localized atomic core electrons.

We propose a technique that directly images the change in the charge density and is thus particularly sensitive to the optically active electrons. The technique, shown in Fig. 1, can monitor simultaneously the rearrangement of the nuclei and the valence electrons in a photochemical reaction. The technique offers the direct observation of transition charge

\footnotetext{
*e-mail: jeremy.rouxel@epfl.ch

**e-mail: smukamel@uci.edu
} 

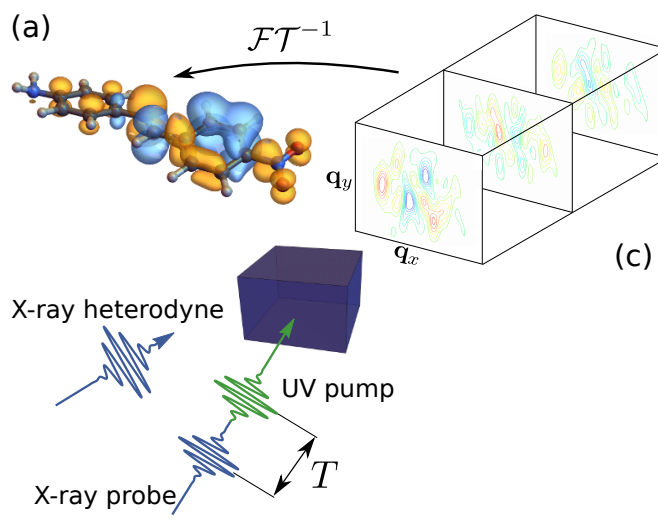

(b)

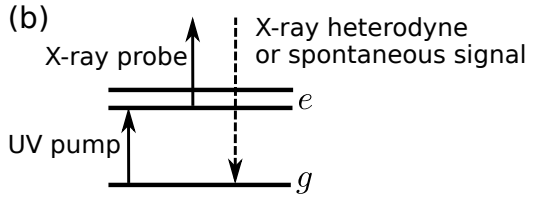

(c)

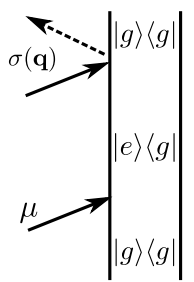

(d)

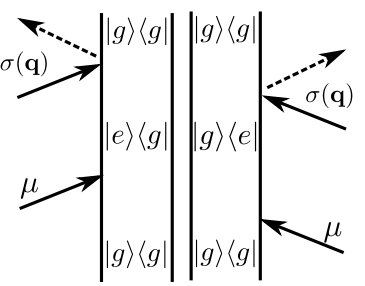

Figure 1. Imaging $\sigma_{g e}(\mathbf{q})$ by the SFG technique. (a) Schematics of the TCD imaging process: A UV pump pulse creates a superposition in the sample and an X-Ray probe pulse creates the diffraction pictures detected with a X-ray heterodyne pulse. The TCD (upper left) can be reconstructed by an inverse Fourier transform. Level scheme (b) for the SFG diffraction signals and corresponding doublesided diagrams for the heterodyne (c) and the homodyne (d) signals. Note that the homodyne signal (d) stems from a two-molecule contribution and thus requires a long-range order in the sample.

densities (TCDs), which contribute to time-resolved diffraction when the molecule is initially prepared in a superposition of states. The charge density is given by

$$
\sigma_{i j}\left(\mathbf{r}_{1}\right)=N \int d \mathbf{r}_{2} \ldots d \mathbf{r}_{N} \Psi_{i}\left(\mathbf{r}_{1} \ldots \mathbf{r}_{N}\right) \Psi_{j}^{*}\left(\mathbf{r}_{1} \ldots \mathbf{r}_{N}\right)
$$

where $\Psi_{i / j}$ are the electronic eigenstates and $\mathbf{r}_{1} . . \mathbf{r}_{N}$ are the electronic coordinates. The TCDs are the off diagonal elements, $\sigma_{i j}(i \neq j)$, which carry valuable chemical information about the molecular orbitals involved in the excitation.

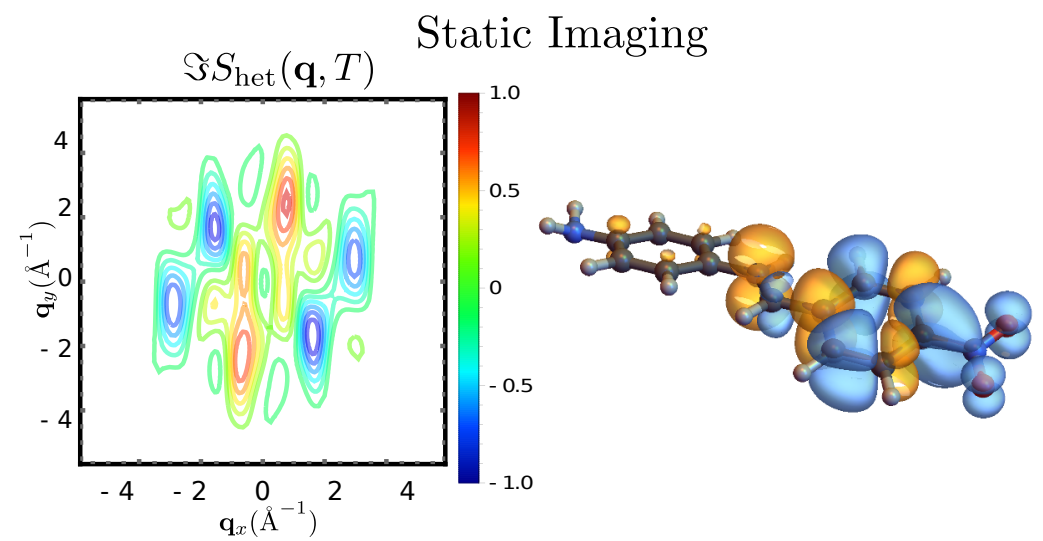

(a)

(b)

Figure 2. Scattering of an X-ray pulse from oriented molecules pumped by a UV pulse that selects the first electronic excited state. (a) SFG diffraction pattern in the $\mathbf{q}_{z}=0$ plane. (b) TCD image obtained by a Fourier transform to real space. 
We have calculated the stimulated SFG diffraction signals for 4-oriented amino4'nitrostilbene. We work in the short time (few femtoseconds) regime where we can neglect nuclear dynamics and radiation damage. The time evolution between the pump and the probe is thus purely electronic. Fig. 2 depicts the static imaging diffraction SFG pattern. The $y$ polarized pump-pulse (Fourier-transform limited with temporal spread (width) $\sigma=5$ fs and frequency $\omega=3.68 \mathrm{eV}$ ) creates a superposition between $g$ and a single state $e$. A 2 fs offresonant X-ray probe pulse is used to probe the superposition. The heterodyne diffraction pattern in Fig. 3(a) carries the phase information of the TCD. The 3D SFG diffraction pattern can be Fourier transformed back into its real-space representation shown in Fig. 3(b), which corresponds to the TCD. The signal oscillation period corresponds to the difference between the pump and the matter transition frequencies. The shape of the signal, i.e. $\sigma_{g e}(\mathbf{r})$, closely resembles a product of the HOMO and LUMO.

\section{References}

[1] J. R. Rouxel, M. Kowalewski, K. Bennett, S. Mukamel, Phys. Rev. Lett. 120, 243902 (2018)

[2] J. R. Rouxel, M. Kowalewski, S. Mukamel, JCTC, 12, 3959-3968 (2016)

[3] J. R. Rouxel, M. Kowalewski, S. Mukamel, J. Phys. Chem. Lett., 9, 3392-3396 (2018) 